\title{
Lentivirus-mediated knockdown of TSP50 suppresses the growth of non-small cell lung cancer cells via G0/G1 phase arrest
}

\author{
WEN-LIANG QIAO ${ }^{1 *}$, HAI-YANG HU ${ }^{1 *}$, BO-WEN SHI ${ }^{1}$, LI-JUAN ZANG ${ }^{2}$, WEI JIN ${ }^{3}$ and QIANG LIN ${ }^{1}$ \\ Departments of ${ }^{1}$ Thoracic Surgery and ${ }^{2}$ Pathology, Shanghai General Hospital, Shanghai Jiao Tong University \\ School of Medicine; ${ }^{3}$ Key Laboratory of Cell Differentiation and Apoptosis of Chinese Ministry of Education, \\ Shanghai Jiao Tong University School of Medicine, Shanghai 200080, P.R. China
}

Received January 11, 2016; Accepted February 18, 2016

DOI: $10.3892 /$ or.2016.4763

\begin{abstract}
Non-small cell lung cancer (NSCLC) as the most frequently diagnosed lethal cancer remains the major cause of overall cancer-related death worldwide. Testes-specific protease 50 (TSP50) has been proved as a critical biomarker in various cancers, and we previously reported that TSP50 protein expression is overexpressed in clinical resected NSCLC tumor tissues and related to poor prognosis in NSCLC patients. Hence, the present study was designed to further investigate the potential oncogenesis mechanism of TSP50 in NSCLC cells. Real-time quantitative PCR, immunohistochemical assay and western blot analysis were used to analyze the TSP50 mRNA and protein expression in 20 NSCLC cases, and TSP50 expression was observed to have high levels in the NSCLC specimens and paired metastatic lymph node tissues when compared to the levels in corresponding normal lung tissues and normal lymph nodes. In the experiments in NSCLC cell lines, lentiviral short hairpin RNA (shRNA) delivery system was applied to knock down TSP50 in 95D cells, and the following investigations revealed that downregulation of TSP50 expression markedly reduced cell proliferation, colony formation and migration ability in vitro. Furthermore, the inhibition of TSP50 induced G0/G1-phase arrest and decreased expression levels of cell cycle relative markers CDK4, CDK6, and CyclinD1 and increased expression of p21 and p53 in 95D cells. In conclusion, this study indicates that TSP50 plays a significant role in NSCLC cell proliferation and may act as a novel oncogene in the development and progression of NSCLC, offering a potential cancer therapeutic target for the treatment of NSCLC.
\end{abstract}

Correspondence to: Dr Qiang Lin, Department of Thoracic Surgery, Shanghai General Hospital, Shanghai Jiao Tong University School of Medicine, 100 Haining Road, Shanghai 200080, P.R. China E-mail: xklinqiang@hotmail.com

*Contributed equally

Key words: TSP50, RNA interference, lentivirus, non-small cell lung cancer, cell cycle, proliferation

\section{Introduction}

Non-small cell lung cancer (NSCLC), accounting for about $80-85 \%$ of lung cancer, is currently the leading cause of cancerrelated mortality in the world (1). Despite growing advance in standard diagnosis and treatment, the expected 5-year survival is only approximately $15 \%$ (2). Patients with early-stage NSCLC could often die from relapse and distant metastasis after curative surgical resection (3), and patients with advancedstage NSCLC received palliative resection or combined with alleviative chemotherapy and radiotherapy frequently fail to approach satisfactory effect (4). In recent years, targeted cancer therapies, as a new class of complementary therapeutical method that specifically target certain molecular pathways leading to cancer phenotypes, demonstrated its advantage in personalized medical treatment in advanced-stage NSCLC (5), while indication of limitations and growing drug resistance of tyrosine kinase inhibitor (TKI) therapy have become critical issues (6). Consequently, better understanding of the molecular mechanisms and identification of tumor related biomarkers are necessary for the development of new effective diagnostic or therapeutic targets for NSCLC.

Testes-specific protease 50 (TSP50), also known as PRSS50 or CT20, encoding a protein homologous to many serine proteases, was isolated from a human testes cDNA library by using a genomic DNA probe (7). Although TSP50 shared two critical catalytic triads, histidine and aspartic acid with traditional serine proteases, the most critical catalytic serine site was substituted with threonine, hence it could be classified as a novel protease (8). Normally, northern blot analysis of multiple human tissue RNA expression panels showed that TSP50 is specifically and strongly expressed in the testes which suggest it might be involved in the human reproductive pathway (9). Reverse transcription-PCR analysis of 18 paired breast cancer tissues first found that the TSP50 gene was differentially expressed in $28 \%$ of the cancer samples (7), and Shan et al confirmed that TSP50 gene activation and overexpression in breast tumors took place in malignant mammary epithelial cells (9). To elucidate the regulatory mechanisms related to differential expression of TSP50 gene in breast cancer, $\mathrm{Xu}$ et al further demonstrated a putative p53-binding site and several Sp1-binding sites in the TSP50 promoter and p53 play a critical role in negatively regulating the TSP50 gene, 
and breast cancer cells containing mutated p53 hence could produce high or low levels of TSP50 transcripts (10); while overexpression of $\mathrm{Sp} 1$ and $\mathrm{C} / \mathrm{EBP} \beta$ transcriptional factors upregulated the activities of the TSP50 promoter in human breast cancer and bFGF mediates TSP50 downregulation by ERK activation, leading to the phosphorylation of Sp1 in this process, which was consistent with the previous research $(11,12)$. Recent results suggested that downregulation of TSP50 induces apoptosis, reduces cell proliferation and colony formation in p19 cells (13). Moreover, knockdown of matrix metalloproteinase-9 (MMP9) resulted in inhibition of cell migration and invasion in vitro, and TSP50 overexpression enhanced expression and secretion of MMP9, hence TSP50 activation of MMP9 is a novel signaling mechanism underlying human breast cancer invasion and metastasis (14).

In addition, TSP50 was also found to be abnormally activated and expressed in laryngocarcinoma and TSP50 knockdown inhibited HEp2 cell proliferation and induced apoptosis in vitro in a NF- $\kappa \mathrm{B}$-mediated pathway $(15,16)$. TSP50 also depends on its threonine protease activity and its interactions with TNF- $\alpha$-induced NF- $\kappa$ B playing an important role in human cervical tumorigenesis (17). Recently, we have reported that TSP50 protein expression is elevated in resected NSCLC tumor tissues as compared with paired non-tumorous tissues using immunohistochemical assay, and found that high levels of TSP50 is related to poor-differentiated tumor, late tumor status and late TNM stage, as well as a reduced disease-free survival and overall survival rate in all NSCLC patients (18). However, the specific molecular mechanisms of TSP50 involved in the development and progression of lung cancer remain largely unknown.

To further investigate the TSP50 expression and its significant role in NSCLC as well as confirm the previous observations, cancer tissue samples with corresponding metastatic lymph nodes from previous NSCLC samples and lung cancer cell lines were detected by immunohistochemical assay, real-time quantitative PCR and western blot analysis. Moreover, we used specific short hairpin RNA (shRNA) to downregulate TSP50 expression in NSCLC cell lines 95D in vitro, and then investigated the proliferation, colony formation, wound healing capacity and cell cycle progression of the cells.

\section{Materials and methods}

Immunohistochemistry. Twenty cases of NSCLC tissues with metastatic lymph nodes and their corresponding adjacent normal tissues were specially selected from 20 patients who underwent surgical resection at the Department of Thoracic Surgery, Shanghai General Hospital, Shanghai Jiao Tong University School of Medicine, between from January 2015 to June 2015. Surgically-removed tumors and matched normal tissues were partially immediately frozen in liquid nitrogen and kept at $-80^{\circ} \mathrm{C}$ until the extraction of the RNA and protein, and partially fixed in neutral-buffered formaldehyde for IHC. These formalin-fixed and paraffin-embedded tissue blocks were cut into 3-4 $\mu \mathrm{m}$ sections and routinely deparaffinized, rehydrated and heated in antigen retrieval with $0.01 \mathrm{~mol} / 1$ citrate buffer solution ( $\mathrm{pH}$ 6.0). Then endogenous peroxidase was blocked in $3 \% \mathrm{H}_{2} \mathrm{O}_{2}$ and non-specific antigen was blocked with $10 \%$ normal goat serum before they were incubated with a rabbit polyclonal antibody against TSP50 (1:150; 12574-1AP, Proteintech) at $4^{\circ} \mathrm{C}$ in a humidified chamber overnight. After being washed with PBS ( $\mathrm{pH} 7.4)$, the slides were hybridized with goat anti-rabbit second antibody conjugated with streptavidin-biotin peroxidase for $20 \mathrm{~min}$ and visualized with 3,3'-diaminobenzidine (DAB), then followed by counterstaining with Mayer's hematoxylin and dehydrated. Each slide was evaluated by two experienced pathologists. The percentage of positive cells in the fields was examined and graded as score 0 (negative), score $1(0-10 \%)$, score 2 (10-50\%), score 3 $(51-75 \%)$ and score $4(75-100 \%)$. The staining intensity of the immunohistochemical staining was graded as follows: 0 (no staining), 1 (weak yellow), 2 (yellow), or 3 (brown). The final score was the sum of the positive cell rate and the intensity of the immunohistochemical staining as $0,1-3,4-5$, or 6-7, which represent negative $(-)$, weakly positive $(+)$, moderately positive $(++)$ and strongly positive $(+++)$, respectively. This study was approved by the Ethics Committee of Shanghai Jiao Tong University School of Medicine and written informed consent was obtained from each patient.

Cell lines and cell culture. Human lung cancer cell lines A549, 95D, Calu-1, H1299, H460, SPC-A-1 and human embryonic kidney cell line HEK293T were all purchased from the Cell Bank of Chinese Academy of Science (Shanghai, China) and maintain in a humidified atmosphere at $37^{\circ} \mathrm{C}$ in $5 \% \mathrm{CO}_{2} .95 \mathrm{D}$, H1299, H460 and SPC-A-1 cells were cultured in RPMI-1640 medium (Hyclone), A549 and 293T cell lines were grown in Dulbecco's modified Eagle's medium (DMEM; Hyclone), and Calu-1 were cultured in McCoy's 5A medium (Sigma, St. Louis, MO, USA). All culture media were supplemented with $10 \%$ heatinactivated fetal bovine serum (FBS, Hyclone), L-glutamine (2 mmol/l) (Life Technologies), penicillin (100 U/ml) and streptomycin $(100 \mu \mathrm{g} / \mathrm{ml})$ (Life Technologies).

Lentivirus vector construction and infection. The lentiviral vector (PLKO.1, Addgene) was used to construct short hairpin RNA (shRNA) specific for TSP50. The TSP50 cDNA sequence was from GenBank with accession number NM_013270.4, the shRNA specific against TSP50 (sh-TSP50) was 5'-CAGTT CTGCTATGAGCTAACTGCTCGAGCAGTTAGCTCATA GCAGAACTTTTTT-3', and a non-specific scramble shRNA sequence (sh-Mock) was 5'-GCGGAGGGTTTGAAAGAA TATCTCGAGATATTCTTTCAAACCCTCCGCTTTTTT-3'. After being sequenced for correct ligation, these recombinant vectors were transfected into HEK293T cells with lentiviral packaging vectors with Lipofectamine 2000 (Invitrogen, Carlsbad, CA, USA) according to the manufacturer's procedure.

The 95D cells were grown in 6-well plates at a density of $5 \times 10^{4}$ cells per well $24 \mathrm{~h}$ before the infection, then the constructed lentiviruses containing TSP50 shRNA and nonsilencing shRNA (sh-Mock) were individually transfected into the 95D cells at a multiplicity of infection (MOI) of 6 with polybrene $(5 \mu \mathrm{g} / \mathrm{ml})$ (Sigma). The efficiency of lentiviruse infection was primarily determined by intensity of green florescence protein (GFP) after $96 \mathrm{~h}$.

RNA extraction and quantitative RT-PCR. Quantitative realtime PCR (qRT-PCR) was used to observe TSP50 expression in 20 pairs of NSCLC cancer and matched metastatic lymph 
Table I. Association of TSP50 expression between NSCLC and non-tumor tissues.

TSP50 protein expression

\begin{tabular}{lcccccr}
\cline { 2 - 6 } Type of tissue & $\begin{array}{c}\text { No. of } \\
\text { cases }\end{array}$ & $\begin{array}{c}\text { Negative } \\
(-)\end{array}$ & $\begin{array}{c}\text { Medium } \\
(+)\end{array}$ & $\begin{array}{c}\text { Positive } \\
(++)\end{array}$ & $\begin{array}{c}\text { Strong Positive } \\
(+++)\end{array}$ & P-value \\
\hline NSCLC tissues & 20 & 4 & 5 & 9 & 2 & $<0.01$ \\
Non-tumor tissues & 20 & 18 & 2 & 0 & 0 & \\
\hline
\end{tabular}

Table II. Association of TSP50 expression between metastatic and non-metastatic lymph node status.

\begin{tabular}{lcccccr}
\hline & & \multicolumn{5}{c}{ TSP50 protein expression } \\
\cline { 3 - 6 } Lymph node tissue & $\begin{array}{c}\text { No. of } \\
\text { cases }\end{array}$ & $\begin{array}{c}\text { Negative } \\
(-)\end{array}$ & $\begin{array}{c}\text { Medium } \\
(+)\end{array}$ & $\begin{array}{c}\text { Positive } \\
(++)\end{array}$ & $\begin{array}{c}\text { Strong Positive } \\
(+++)\end{array}$ & P-value \\
\hline Metastatic lymph node & 20 & 9 & 5 & 6 & 0 & $<0.05$ \\
Non-metastatic lymph node & 20 & 11 & 9 & 0 & 0 & \\
\hline
\end{tabular}

node specimens (kept in liquid nitrogen) and knockdown efficiency in 95D cells. Total RNA of specimens and cells harvested and washed with PBS were extracted with TRIzol reagent (Invitrogen). Samples with 260/280 values of 1.8-2.0 were considered for further research. cDNA was then reverse transcribed with oligodT using M-MLV reverse transcriptase (Thermo Fisher Scientific, Rockford, IL, USA). Quantitative PCR was performed with Maxima SYBR Green qPCR Master Mixes (Thermo Fisher Scientific) in an ABI 7300 system (Applied Biosystem, Foster City, CA, USA), and approximate $20 \mu \mathrm{l}$ of qRT-PCR mixtures containing were 2X SYBR Premix Ex Taq $10 \mu \mathrm{l}$, forward primers $0.5 \mu 1$ $(2.5 \mu \mathrm{M})$, reverse primers $0.5 \mu \mathrm{l}(2.5 \mu \mathrm{M})$, cDNA $5 \mu \mathrm{l}$, and $\mathrm{ddH}_{2} \mathrm{O} 4.5 \mu \mathrm{l}$. All $40 \mathrm{PCR}$ cycles were run using the following cycling parameters, predegeneration for $1 \mathrm{~min}$ at $95^{\circ} \mathrm{C}$, denaturation for $5 \mathrm{sec}$ at $95^{\circ} \mathrm{C}$, and extension for $30 \mathrm{sec}$ at $60^{\circ} \mathrm{C}$. The primer sequences for PCR amplification of the TSP50 were F: 5'-GCTCCTGTGGCTTTTCCTAC-3' and R: 5'-CGGAGGCGGTCTGCGTCAT-3'. The primer sequences for $\beta$-actin were F: 5'-GTGGACATCCGCAAAGAC-3' and R: 5'-AAAGGGTGTAACGCAACTA-3'. The fold change of TSP50 mRNA expression was calculated by the $2^{-\Delta \Delta \mathrm{Ct}}$ method, using $\beta$-actin mRNA expression level for normalization. All experiments were repeated in triplicate.

Western blot analysis. The liquid nitrogen-conserved NSCLC tissues were homogenized and lysed in the RIPA buffer on ice. Protein concentrations were determined by the Bradford method using bovine serum albumin as the standard. The 95D cells were collected 7 days after lentivirus infection and washed with ice-cold PBS 2 times then harvested in RIPA lysis buffer $(150 \mathrm{mM}$ Tris- $\mathrm{HCl}, 50 \mathrm{mM} \mathrm{NaCl}, 1 \% \mathrm{NP}-40$, $0.1 \%$ Tween-20) with freshly added protease inhibitor cocktail (Sigma). Equal amount of protein $(30 \mu \mathrm{g})$ were mixed with $5 \mathrm{X}$ loading buffer, boiled for $5 \mathrm{~min}$, and loaded into a $10 \%$ sodium dodecyl sulfate-polyacrylamide (SDS) gel electrophoresis and transferred onto a polyvinylidene fluoride (PVDF, Millipore, Bedford, MA, USA) membrane by electroblotting for $90 \mathrm{~min}$. Non-specific sites on membranes were then blocked with 5\% skim-milk for $1 \mathrm{~h}$ then incubated with primary antibodies antiTSP50, anti-p21 and anti-CDK6 (Abcam, Cambridge, MA, USA); anti-CDK4, anti-CyclinD1, anti-GAPDH and p53 (Cell Signaling Technology, Danvers, MA, USA) overnight at $4^{\circ} \mathrm{C}$, and then probed with goat anti-rabbit HRP-linked secondary antibody (Santa Cruz Biotechnology, Santa Cruz, CA, USA). Immunoreactive bands were identified using the enhanced chemiluminescence (ECL) system (Bio-Rad Laboratories, Richmond, CA, USA) following the manufacturer's instructions. GAPDH was used as a loading control.

Cell viability assay. Transfected cell proliferation was determined using an MTT assay. After lentivirus infection, 95D cells were placed with $100 \mu \mathrm{l}$ at a density of $2 \times 10^{3}$ cells/well in 96-well microtitre plates. During the following 5 days, the absorbance data of one specified plate was analyzed each day, and the cells in the rest of the plates were cultured continuously. A total of $20 \mu 1$ MTT [3-(4,5-dimethylthiazol-2-yl)-2, 5-diphenyltetrazolium bromide] $(5.0 \mathrm{mg} / \mathrm{ml})$ was added into each well and incubated with cells at $37^{\circ} \mathrm{C}$ for $4 \mathrm{~h}$, then the reaction was terminated by adding $100 \mu \mathrm{l}$ acidic isopropanol (5\% isopropanol, $10 \% \mathrm{SDS}$, and $0.01 \mathrm{~mol} / 1 \mathrm{HCl}$ ). Fast colorimetric assay for cellular growth was measured the next day by a microplate reader (Bio-Rad Laboratories) in absorbance at wavelength $595 \mathrm{~nm}$. The results were plotted as the mean \pm standard deviations (SD) of five determinations.

Colony formation assay. Three kinds of 95D cells were plated onto 6-well plates with $2 \mathrm{ml}$ at a density of 500 cells per well for 8 days with the culture medium changed every 3 days. When most of the colonies were $>50$ cells, adherent cells were gently washed with $\mathrm{ddH}_{2} \mathrm{O} 2$ times and fixed in $800 \mu \mathrm{l}$ fresh prepared $4 \%$ paraformaldehyde at room temperature for $15 \mathrm{~min}$. The 

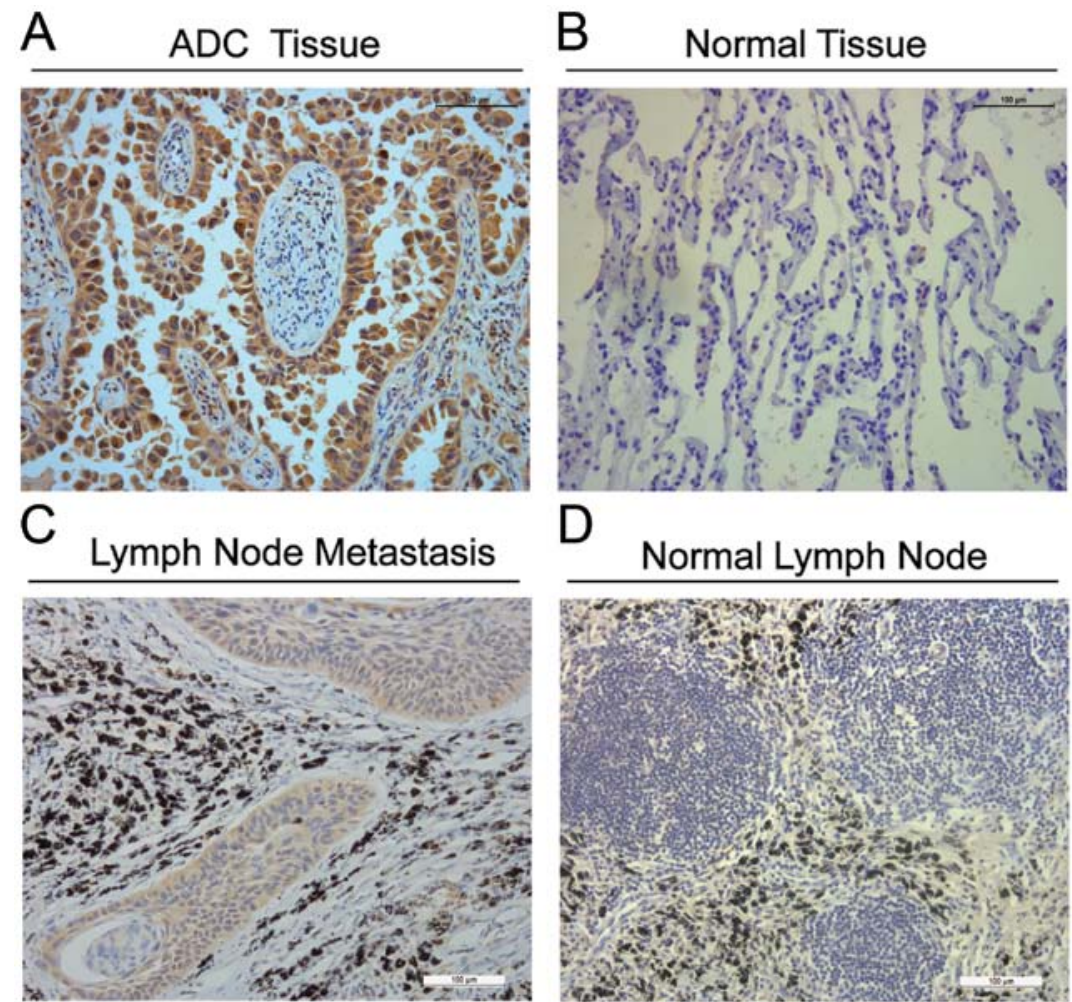

Figure 1. Representative images of TSP50 immunohistochemical overexpression in human NSCLC and metastatic lymph node tissues (x200 magnification). (A) Strong positive TSP50 expression in the cytoplasms of lung adenocarcinoma (ADC). (B) Negative TSP50 expression in normal lung tissues. (C) Positive TSP50 immunohistochemical expression in metastatic lymph node tissues of lung squamous cell carcinoma. (D) Negative TSP50 expression in non-metastatic lymph node tissues. NSCLC, non-small cell lung cancer; TSP50, testes-specific protease 50.

cells were then stained with Giemsa (Sigma-Aldrich) for 10 min and washed with $\mathrm{ddH}_{2} \mathrm{O} 3$ times. Colonies with $>50$ cells were photographed with fluorescence microscope. Three independent experiments were repeated.

Wound healing assay. To measure the invasion ability of cells, each group of cells were grown in 96-well microtitre plates incubated with RPMI-1640/fetal bovine serum (10\%) in a volume of $100 \mu \mathrm{l}$ per well overnight. When primary cultures became confluent and a wound was created with a sterile pipette tip. After being washed with PBS 2 times, 2\% RPMI-1640/fetal bovine serum was added into plates and each group of cells were fixed and observed by microscopy at the time point of $24 \mathrm{~h}$. The experiment was independently performed for three times.

Cell cycle analysis. The role of TSP50 silencing on 95D cell cycle distribution was evaluated by flow cytometry with propidium iodide (PI, Sigma) staining. Briefly, 95D cells were seeded in $6-\mathrm{cm}$ dishes with $5 \mathrm{ml}$ volume at a density of $1 \times 10^{6}$ cells/well after infected with indicated virus for 6 days, then cells were harvested with trypsinization after $48 \mathrm{~h}$, washed with ice-cold PBS, and fixed in $75 \%$ ice-cold ethanol overnight at $4^{\circ} \mathrm{C}$. After the ethanol was removed by centrifugation, cells were washed with PBS two times and incubated with PI $(50 \mu \mathrm{g} / \mathrm{ml}$, Sigma) solution containing $100 \mu \mathrm{g} / \mathrm{ml}$ of ribonuclease A (Sigma) at room temperature for $30 \mathrm{~min}$. DNA content of all stained cells was then analyzed on flow cytometer (BD Biosciences, Franklin Lakes, NJ, USA). Experiments were performed in triplicate.

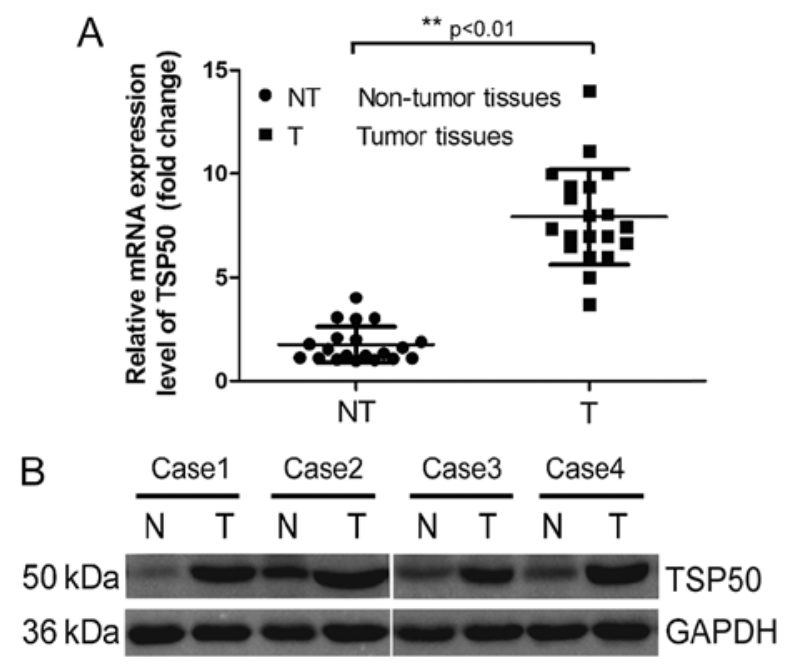

Figure 2. Expression of TSP50 in NSCLC samples (T) and adjacent nontumor tissue (NT) samples. (A) Quantitative real-time PCR analysis of TSP50 mRNA expression in 20 paired fresh NSCLC and non-tumor tissues, and fold change of the TSP50 was calculated by the equation $2^{-\Delta \Delta C t}$. All experiments were performed in triplicate. (B) The representative different expressions of TSP50 protein in 4 matched NSCLC tissues by western blot analysis. TSP50 was detected with anti-TSP50 antibody at 1:1,000 dilution. GAPDH was used as internal loading control.

Statistical analysis. Results were compared using the Student's t-test and considered statistically significant at a P-value of $<0.05$. Data were expressed as mean \pm SD and all experiments were carried out independently at least three times. 


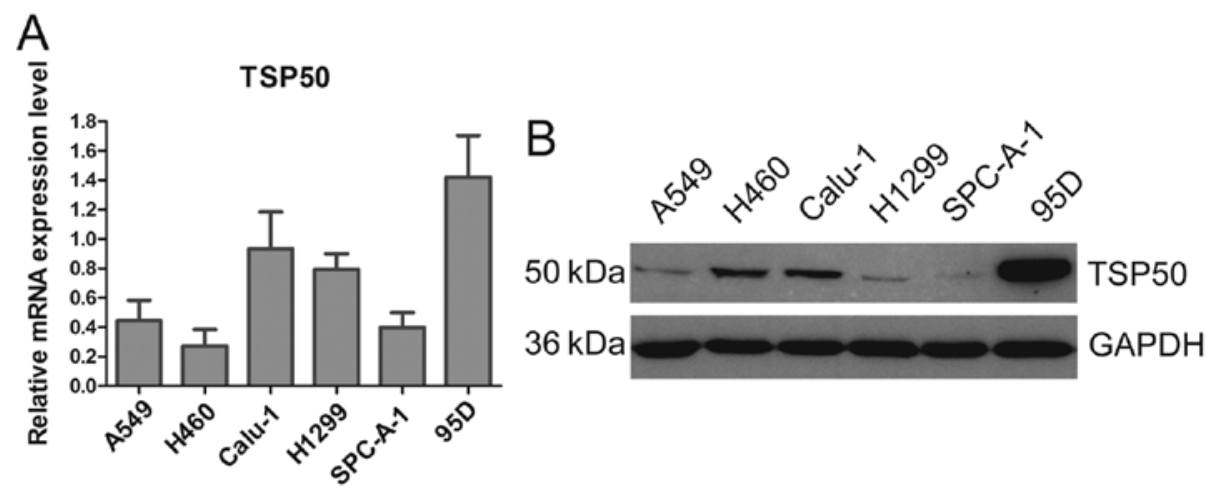

Figure 3. Relative gene and protein expression of TSP50 in A549, H460, Calu-1, H1299, SPC-A-1 and 95D lung cancer cell lines. (A) The mRNA levels of TSP50 were detected by quantitative real-time PCR in six lung cancer cell lines. $\beta$-actin was used as normalized control. (B) Western blot analysis was performed to examine TSP50 protein expression in six lung cancer cell lines. GAPDH was used as the loading control. Data are presented as the mean \pm SD of three independent experiments.

A

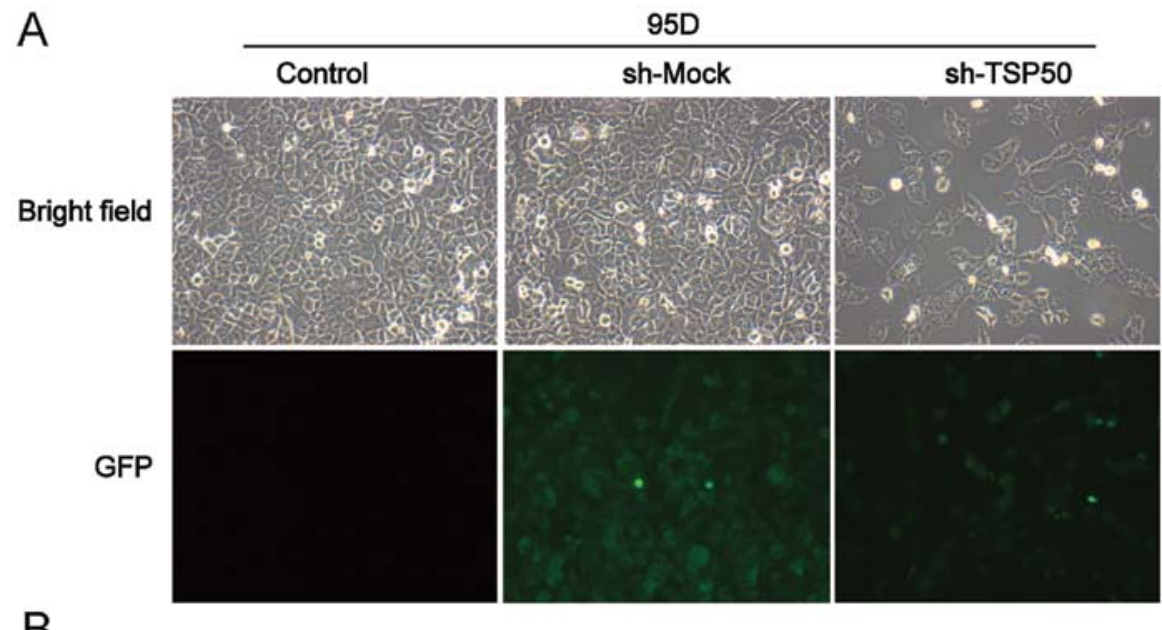

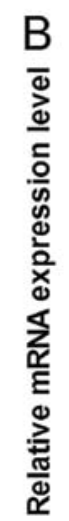

Bright field
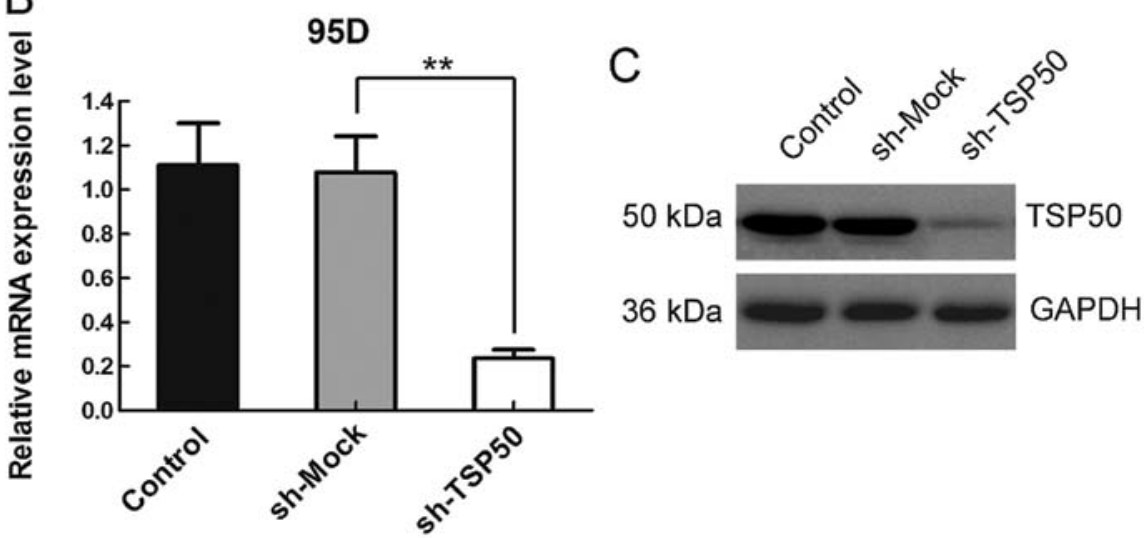

Figure 4. Detection of the knockdown efficiency after lentivirus-mediated silencing of TSP50 by RNA interference (RNAi). (A) TSP50 shRNA lentivirus and the non-silencing shRNA lentivirus expressing green florescence protein (GFP) were constructed and transfected into 95D cells. GFP expression was detected in Control, sh-Mock and sh-TSP50 infected 95D cells after $96 \mathrm{~h}$ of infection. (B) TSP50 mRNA level was significantly decreased in the sh-TSP50 group by quantitative real-time PCR (** $\mathrm{P}<0.01)$. $\beta$-actin was used as the internal control. (C) TSP50 protein level was downregulated remarkably in the sh-TSP50 group by western blot analysis. GAPDH was used as the internal loading control. Data are presented as the mean \pm SD of three independent experiments.

\section{Results}

High levels of TSP50 expression in NSCLC and metastatic lymph nodes. Overall results of the TSP50 protein in NSCLC and metastatic lymph node tissues is summarized in Tables I and II, positive staining of TSP50 was detected in the cytoplasms of adenocarcinoma and squamous cell carcinoma tissues, significant correlation was observed both in between NSCLC and non-cancer tissues, and between the metastatic lymph node and non-metastatic lymph node subgroups, respectively (Fig. 1). In addition, the RT-qPCR data confirmed increased levels of TSP50 mRNA expression in the 20 NSCLC specimens compared with the adjacent non-tumor tissues (Fig. 2A). Consistent with the mRNA level, western 
A
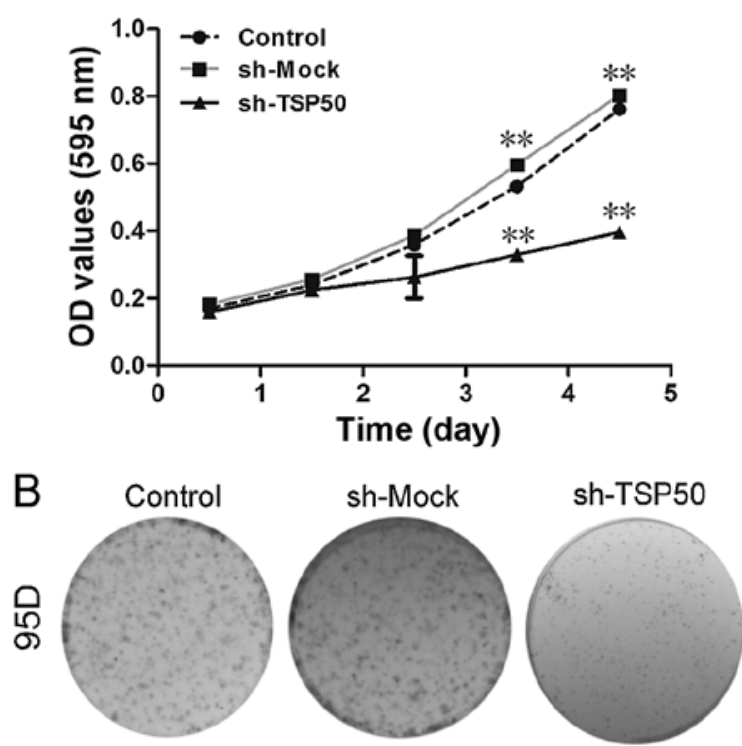

C

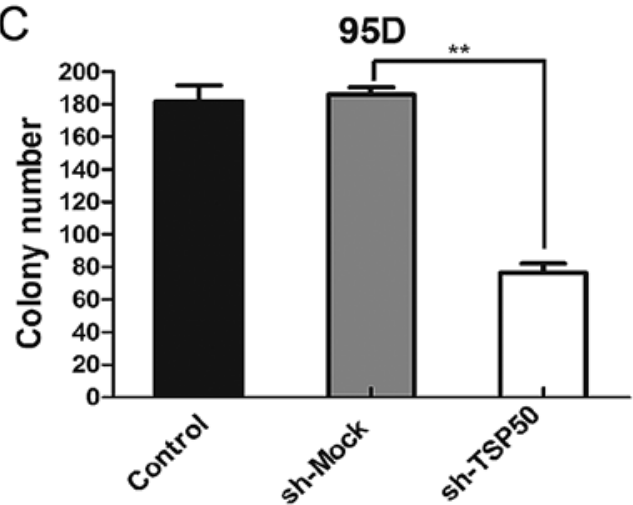

Figure 5. The role of TSP50 knockdown on cell proliferation, colony formation and migration ability in 95D cells. (A) Effect of TSP50 silencing on cell proliferation. Methylthiazol tetrazolium (MTT) assay showed that the growth curve was significantly inhibited in 95D cells after TSP50 knockdown (B) Effect of TSP50 knockdown on the colony formation. Representative photomicrographs of Giemsa-stained colonies of 95D cells growing in culture plates for 8 days after infection. Obvious decrease was observed in the total number of colonies formed in 95D cells transfected with sh-TSP50 against control groups. (C) Colonies consisting of more than 50 cells in each well were counted. Comparing with the number of colonies in control groups, the number of colonies in the sh-TSP50 group was significantly reduced. Data are expressed as mean $\pm \mathrm{SD}$ of three independent experiments. ${ }^{* *} \mathrm{P}<0.01$.

blot analysis also showed that $13(65 \%)$ NSCLC samples had significantly higher TSP50 protein levels than that of the adjacent non-tumor tissues (Fig. 2B).

Knockdown of TSP50 with shRNA lentiviruses systems in 95D cells. TSP50 in six NSCLC cell lines including A549, H460, Calu-1, H1299, SPC-A-1 and 95D lung cancer cells was evaluated by real-time PCR and western blot analysis. As shown in Fig. 3A and B, 95D cells showed the highest transcription level of TSP50 and indicated highest levels of TSP50 protein expression, thus they were chosen to further investigate the potential biological function of TSP50 in NSCLC.

shRNA against TSP50 (shTSP50) and non-specific scramble shRNA sequence (sh-Mock) were cloned into a lentiviral vector expressing GFP which were further infected into 95D cells, respectively, and GFP expression was generated and examined with fluorescence microscopy to assess the lentiviral infection efficiency. The infection efficiency of lentivirus was $>90 \%$ after $96 \mathrm{~h}$ of infection (Fig. 4A). In addition, the knockdown efficiency was further confirmed by qRT-PCR and western blot analysis. As shown in Fig. 4B, the qRT-PCR assay revealed that the TSP50 mRNA level was reduced by $80 \%$ in the sh-TSP50 group in comparison with that in the sh-Mock and Control group. The TSP50 protein expression was also markedly downregulated in the shTSP50 group in contrast with the other two groups (Fig. 4C). Therefore, the recombinant RNA interference lentivirus system was able to efficiently knock down TSP50 expression in 95D cells.

Silencing of TSP50 reduces cell proliferation and colony formation. To further analyze the biological function of TSP50 in the development and progression of NSCLC, we performed MTT and colony formation after infecting 95D cells with shRNA and sh-Mock controls. As shown in Fig. 5A, MTT cell proliferation assay indicates that the growth curves of sh-TSP50 group was significantly inhibited and the proliferation rate of $95 \mathrm{D}$ cells was reduced by 48.6 and $53.3 \%$ on the 4th and 5th day, respectively, compared to that of control groups. However, there was no difference between sh-Mock infected and non-infected 95D cells. This finding indicates that the knockdown of TSP50 markedly decreased the cell proliferative ability in NSCLC cancer cells.

To further investigate and confirm the effect of TSP50 silencing on the proliferation of 95D cells, the colony formation assay was performed. As depicted in Fig. 5B, 95D cells infected with sh-TSP50 formed smaller colony size and lower colony density compared with controls, and the number of colonies was correspondingly decreased by 61.2 and $62.6 \%$ after shTSP50 infection, respectively (Fig. 5C, $\mathrm{P}<0.01$ ). The results further demonstrated that silencing of TSP50 could significantly suppress the colony-forming ability of NSCLC cells. Collectively, these data suggest that TSP50 may act as a positive regulator of cell growth and plays a critical role in NSCLC carcinogenesis.

Suppression of $95 D$ cell migration in vitro by TSP50 knockdown. We further evaluated the effect of TSP50 on the migratory ability of the 95D cells by wounded healing assay after lentivirus infection with sh-TSP50 and sh-Mock controls. We observed that migratory ability of 95 cells in sh-TSP50 group was much weaker than those in sh-Mock group at the $48 \mathrm{~h}$ point after infection $(\mathrm{P}<0.05)$ (Fig. 6). Therefore, these results preliminarily indicate that TSP50 may play a key role in the migration of $95 \mathrm{D}$ cells.

Suppression of TSP50 induces G0/G1 phase arrest of 95D cells. In view of the findings that knockdown of TSP50 in 95D cells markedly inhibits cell proliferation and decrease cell colony formation ability, we further performed cell cycle analysis to investigate the mechanism underlying the inhibition of 95D cell growth by flow cytometry analysis after PI staining. As shown in Fig. 7A and B, a marked increase in the G0/G1 phase cell population was observed in the sh-TSP50infected 95D cells accompanied by a decrease in the $\mathrm{S}$ and $\mathrm{G} 2 / \mathrm{M}$ phase cell population, when in contrast with these 


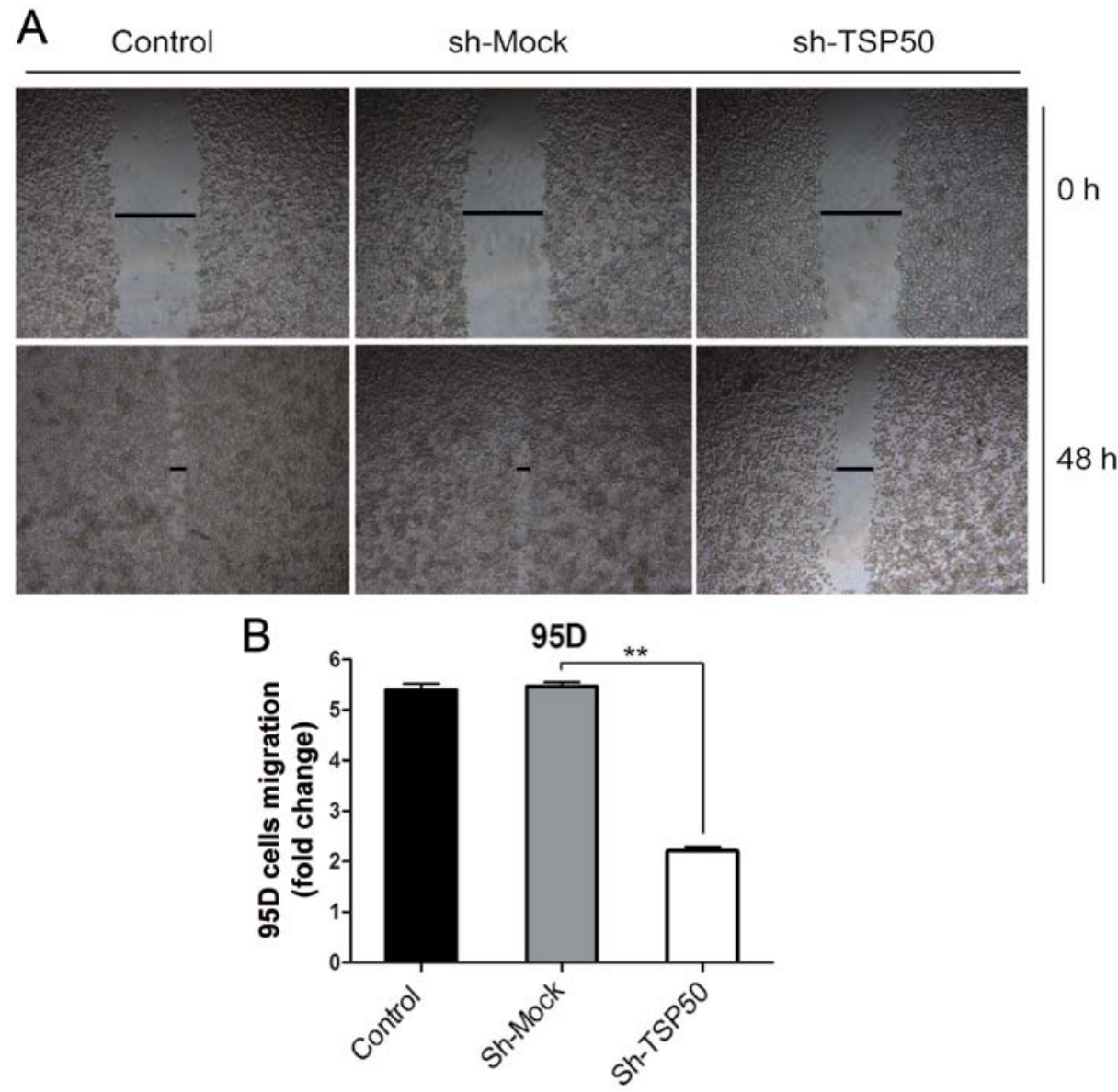

Figure 6. Effect of TSP50 knockdown on cell migration detected by wound healing assay. (A) The 95D lung cancer cells transfected with sh-TSP50 showed remarkably decreased migratory ability compared with the sh-Mock and Control groups under the microscope. (B) Cell migration of 95D lung cancer cells for $48 \mathrm{~h}$ after transfection, and fold change of migration is presented as a value relative to the distance moved by the cell monolayer in a scrambled control. Data are expressed as the mean $\pm \mathrm{SD}$ of three independent experiments. ${ }^{* *} \mathrm{P}<0.01$.

percentages in the sh-Mock infected 95D cells; however, no significant difference in percentage of cells was observed between the groups of sh-Mock and Control during G0/G1, S, and $\mathrm{G} 2 / \mathrm{M}$ phases. Further, we detected the protein levels of G0/G1-associated genes including CyclinD1, CDK4, CDK6, p21 and p53 by western blot analysis. As shown in Fig. 7C, knockdown of TSP50 resulted in decrease in CDK4, CDK6 and cyclinD1 expression, and increase in p21 and p53 gene expression. Consequently, our results suggested that TSP50 silencing was able to inhibit the growth of NSCLC cells via inducing G0/G1 cell cycle arrest.

\section{Discussion}

NSCLC as the predominant form of lung cancer accounting for the majority of lung cancer associated deaths worldwide (1). There has been an unceasing and positive effort to improve prognosis of NSCLC, and novel treatment strategies have arisen from the research of the molecular and cellular biology of NSCLC. Here, we investigated the functional role of TSP50 gene in NSCLC and its potential application as a therapeutic target. Firstly, high levels of TSP50 expression in NSCLC tissues was detected at both the RNA and protein levels and in corresponding metastatic lymph nodes, these results were in accordance with a previous study and suggest that TSP50 may be related to both tumorigenesis and development of NSCLC, hence it could be a potential therapeutic target $(19,20)$.

TSP50 as a testis-specific gene re-expressed in different types of malignant somatic cells indicate the TSP50 gene may be an oncogene. Thus, to further investigate the functional significance of TSP50 and evaluate its application prospect of targeted gene therapy in NSCLC cells, we conducted an in vitro experiment using lentivirus shRNA system that steadily and effectively knocked down the expression of TSP50, and observed the subsequent effect of TSP50 silencing on progression in $95 \mathrm{D}$ cells. To our knowledge, this is the first study to report TSP50 expression in NSCLC cells and investigate the role in functional phenotype of NSCLC cells. qRT-PCR and western blot analysis showed targeting gene was inhibited effectively, hence ensuring the credibility of the following assays. As expected, knockdown of TSP50 significantly reduced cell proliferation, colony formation, migration ability and arrested cell cycle in 95D cell lines. These results were coincident with recent reports that downregulation of TSP50 expression inhibited the growth of breast cancer and laryngocarcinoma cells $(14,16)$.

Additionally, TSP50 as a testis-specific gene overexpressed in various malignant cells was considered as an oncogenic cancer/testis (CT) antigen family member, also including SSX-2, MAGE-A1, NY-ESO-1 and others (21). Importantly, 
A $95 \mathrm{D}$
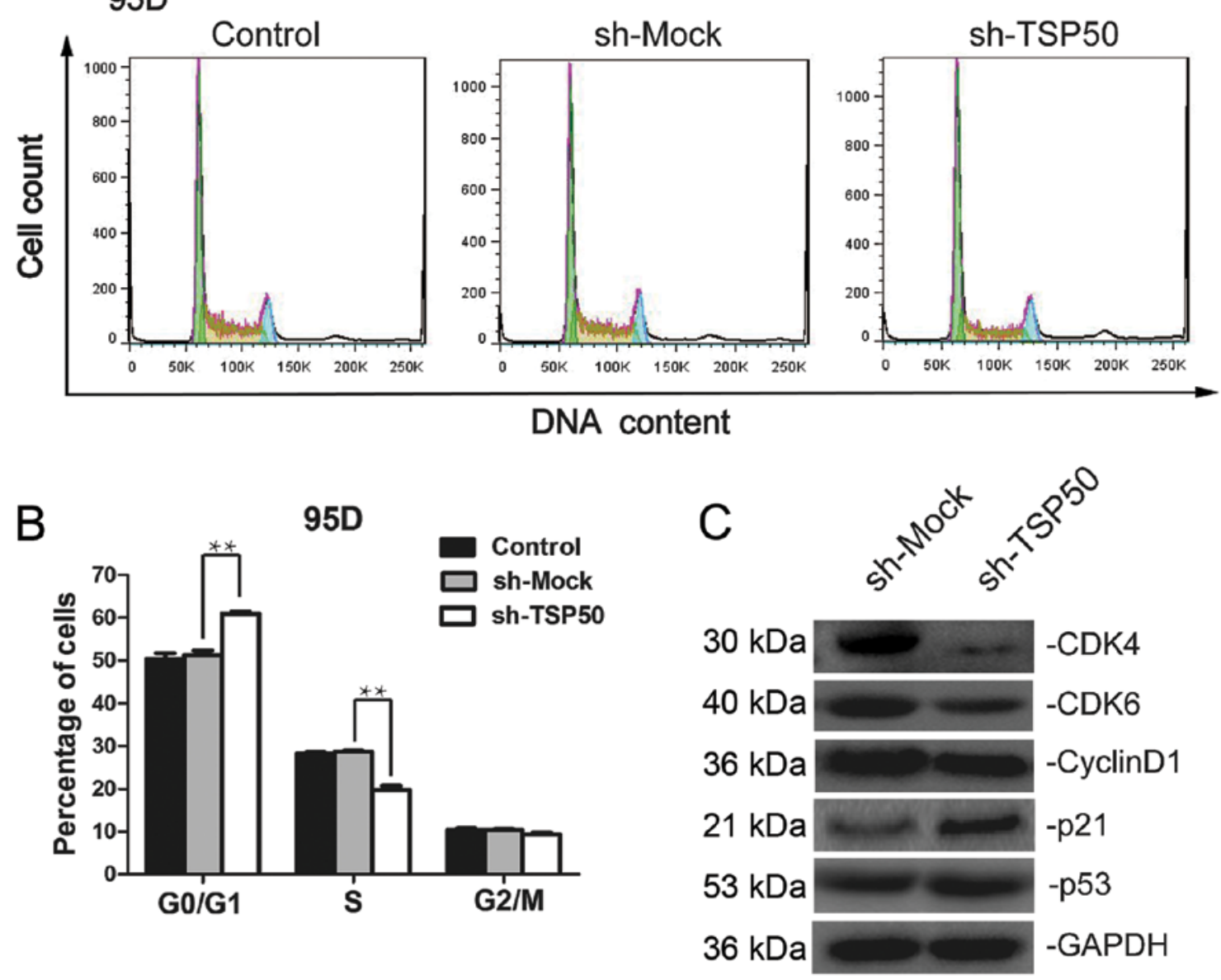

Figure 7. Effect of TSP50 knockdown on the cell cycle progression of 95D cells. (A) Cell cycle distribution patterns of 95D cells in Control, sh-Mock, and sh-TSP50 groups was analyzed using flow cytometry. (B) Knockdown of TSP50 suppressed cell growth at G0/G1 phase transition in 95D cells. (C) Western blot analysis showed that the protein expression levels of cell cycle genes CDK4, CDK6, and cyclinD1 were decreased, while p21 and p53 were increased in the sh-TSP50 group. GAPDH was used as internal loading control. Experiments were performed in triplicate. ${ }^{* *} \mathrm{P}<0.01$.

many CT antigens have been shown to support cancer cell growth (22). Recent observations indicated that knockdown of the CT antigen SSX2 in melanoma cells significantly decrease cellular proliferation ability (23), GAGE7 played a preventing role in response to different types of apoptotic stimuli $(24,25)$, and silencing of PAGE4 could attenuate tumor growth by inducing cell death in vivo (26). Consequently, our data indicate similar features of TSP50 with other CT antigens from another angle, further demonstrating TSP50 plays a crucial role in tumorigenesis of NSCLC. In addition, migration ability is the essential processes towards metastasis of cancer, combined with the high levels of TSP50 expression in the paired metastatic lymph nodes, we believe that the effects of TSP50 may be closely related with the metastasis process of NSCLC.

Noteworthy, our data first revealed that TSP50 silencing had an inhibitory effect on cell cycle progression of 95D cell lines via G0/G1 phase, which has not been reported in other cancer cell lines. To clarify the possible molecular mechanism involved in 95D cell proliferation, we detected modifications of some crucial checkpoint and regulatory molecules related to G0/G1 to $\mathrm{S}$ phase in 95D cells with TSP50 knockdown. Previous studies have confirmed cell cycle progression is positively regulated by a series of Cyclins and CDKs (27).
CyclinD1 is regarded as one of the significant oncogenic drivers in cancer cells and is the most distinctive G1-phase cyclin (28), and CDK4/6 along with its activating cyclin partner D1 has a critical role in cell cycle control (29).

CyclinD1 binding to CDK4/6 forms the active complex of CyclinD1-CDK4/6, which could further assemble cyclin and CDK subunits and activate specific gene expression required for $\mathrm{G} 0 / \mathrm{G} 1$ to $\mathrm{S}$ phase progression $(30,31)$. However, the CDK inhibitor family members including p21, p16 and p27 have a negative regulatory role in this process $(32,33)$. For instance, p21 could inactivate and degrade the CyclinD1-CDK4/6 compound and bind and inhibit the proliferating cell nuclear antigen to arrest the G0/G1 to $\mathrm{S}$ phase transition (34). Besides, p53 known as a 'superstar' marker could be activated by DNA damage checkpoint kinases to simultaneously control the G1/S and $\mathrm{G} 2 / \mathrm{M}$ cell cycle checkpoints, with the p53 binding sites in p21 promoter, p21 gene could be activated and regulated by p53 gene at the transcriptional level $(35,36)$. In our research, we found that knockdown of TSP50 reduced the levels of CDK4, CDK6 and cyclinD1 expression, and increased the level of p21 and p53 expression in 95D cells, which indicated that TSP50 knockdown induced G0/G1 phase arrest partly via suppression of these related cell cycle regulators. Besides, the trend of p53 change in sh-TSP50 and sh-Mock 95D cells according to a 
previous study showing TSP50 is a downstream protein of p53 for its promoter containing a putative p53-binding site and it was negatively regulated by the p53 transgene in diverse types of cell lines (10).

$\mathrm{CT}$ antigens are immunogenic proteins expressed in normal testis and in different types of tumors. Because of their immunogenicity and testis-restricted normal tissue expression, $\mathrm{CT}$ antigens are prioritized targets for antigen-specific cancer immunotherapy and the identification of novel CT antigens is a prerequisite for the development of cancer vaccines $(37,38)$. Moreover, Kim et al demonstrated CT antigens are potential candidates in lung cancer patients for polyvalent immunotherapy (21). Since our data have proved that TSP50 can be used as a prognostic or predictive biomarker in NSCLC, further immunotherapy of TSP50 in NSCLC as a supplement to conventional treatment will be conducted.

In summary, this is the first study discussing the possible role of TSP50 in NSCLC cell growth and migration ability, which provide further specific molecular mechanisms to our previous study indicating the considerable potential of TSP50 to be a new therapeutic target for the treatment of NSCLC.

\section{Acknowledgements}

This study was supported by grants from The National Natural Science Foundation of China (General Program) (81372521). We are grateful to all the Staff of the Experimental Center of Shanghai General Hospital for their sincere help and technical support.

\section{References}

1. Molina JR, Yang P, Cassivi SD, Schild SE and Adjei AA Non-small cell lung cancer: Epidemiology, risk factors, treatment, and survivorship. Mayo Clin Proc 83: 584-594, 2008.

2. Katzel JA, Fanucchi MP and Li Z: Recent advances of novel targeted therapy in non-small cell lung cancer. J Hematol Oncol 2: $2,2009$.

3. Gilmore DM, Khullar OV, Jaklitsch MT, Chirieac LR, Frangioni JV and Colson YL: Identification of metastatic nodal disease in a phase 1 dose-escalation trial of intraoperative sentinel lymph node mapping in non-small cell lung cancer using near-infrared imaging. J Thorac Cardiovasc Surg 146: 562-570, 2013.

4. Kumarakulasinghe NB, van Zanwijk N and Soo RA: Molecular targeted therapy in the treatment of advanced stage non-small cell lung cancer (NSCLC). Respirology 20: 370-378, 2015.

5. Fukui T, Taniguchi T, Kawaguchi K, Fukumoto K, Nakamura S, Sakao Y and Yokoi K: Comparisons of the clinicopathological features and survival outcomes between lung cancer patients with adenocarcinoma and squamous cell carcinoma. Gen Thorac Cardiovasc Surg 63: 507-513, 2015.

6. Yang YC, Cheng TY, Huang SM, Su CY, Yang PW, Lee JM, Chen CK, Hsiao M, Hua KT and Kuo ML: Cytosolic PKM2 stabilizes mutant EGFR protein expression through regulating HSP90-EGFR association. Oncogene: Oct 26, 2015 (Epub ahead of print).

7. Yuan L, Shan J, De Risi D, Broome J, Lovecchio J, Gal D, Vinciguerra V and Xu HP: Isolation of a novel gene, TSP50, by a hypomethylated DNA fragment in human breast cancer. Cancer Res 59: 3215-3221, 1999.

8. Wei J, Liu Y, Yang S, Xu J, Kong H, Han B, Bao Y, Wu Y, Yin W, $\mathrm{Li} \mathrm{W}$, et al: Screening of single-chain variable fragments against TSP50 from a phage display antibody library and their expression as soluble proteins. J Biomol Screen 11: 546-552, 2006.

9. Shan J, Yuan L, Xiao Q, Chiorazzi N, Budman D, Teichberg S and $\mathrm{Xu}$ HP: TSP50, a possible protease in human testes, is activated in breast cancer epithelial cells. Cancer Res 62: 290-294, 2002.
10. Xu H, Shan J, Jurukovski V, Yuan L, Li J and Tian K: TSP50 encodes a testis-specific protease and is negatively regulated by p53. Cancer Res 67: 1239-1245, 2007.

11. Wang M, Bao YL, Wu Y, Yu CL, Meng X, Xu HP and Li YX: Identification and characterization of the human testes-specific protease 50 gene promoter. DNA Cell Biol 27: 307-314, 2008

12. Wang M, Bao YL, Wu Y, Yu CL, Meng XY, Huang YX, Sun Y, Zheng LH and Li YX: Basic FGF downregulates TSP50 expression via the ERK/Sp1 pathway. J Cell Biochem 111: 75-81, 2010.

13. Zhou L, Bao YL, Zhang Y, Wu Y, Yu CL, Huang YX, Sun Y, Zheng LH and Li YX: Knockdown of TSP50 inhibits cell proliferation and induces apoptosis in P19 cells. IUBMB Life 62: 825-832, 2010.

14. Song ZB, Ni JS, Wu P, Bao YL, Liu T, Li M, Fan C, Zhang WJ, Sun LG, Huang YX, et al: Testes-specific protease 50 promotes cell invasion and metastasis by increasing NF-kappaB-dependent matrix metalloproteinase-9 expression. Cell Death Dis 6: e1703, 2015.

15. Liu YL and Sun YN: Down-regulation of testes-specific protease 50 induces apoptosis in human laryngocarcinoma HEp2 cells in a NF-кB-mediated pathway. Mol Biol Rep 41: 7743-7747, 2014.

16. Zhang X: Depression of testes-specific protease 50 (TSP50) inhibits cell proliferation and induces apoptosis in laryngocarcinoma. Tumour Biol 35: 10781-10788, 2014.

17. Yuan J, Wu C, Huang M, Zhou J, Ben W and Zhang G: TSP50 depends on its threonine protease activity and its interactions with TNF- $\alpha$-induced NF- $\kappa$ B for its role in human cervical tumorigenesis. Cell Biochem Biophys 71: 891-896, 2015.

18. Qiao WL, Shi BW, Han YD, Tang HM, Lin J, Hu HY and Lin Q: Testes-specific protease 50 as an independent risk factor for poor prognosis in patients with non-small cell lung cancer. Oncol Lett (In press).

19. Liu F, Cao Q, Liu N, Li C, You C, Liu C, Xue L and Luo R: Overexpression of testes-specific protease 50 (tsp50) predicts poor prognosis in patients with gastric cancer. Gastroenterol Res Pract 2014: 498246, 2014

20. Zheng L, Xie G, Duan G, Yan X and Li Q: High expression of testes-specific protease 50 is associated with poor prognosis in colorectal carcinoma. PLoS One 6: e22203, 2011.

21. Kim YD, Park HR, Song MH, Shin DH, Lee CH, Lee MK and Lee SY: Pattern of cancer/testis antigen expression in lung cancer patients. Int J Mol Med 29: 656-662, 2012.

22. Gjerstorff MF, Andersen MH and Ditzel HJ: Oncogenic cancer/ testis antigens: Prime candidates for immunotherapy. Oncotarget 6: 15772-15787, 2015.

23. Greve KB, Lindgreen JN, Terp MG, Pedersen CB, Schmidt S, Mollenhauer J, Kristensen SB, Andersen RS, Relster MM, Ditzel HJ, et al: Ectopic expression of cancer/testis antigen SSX2 induces DNA damage and promotes genomic instability. Mol Oncol 9: 437-449, 2015.

24. Cilensek ZM, Yehiely F, Kular RK and Deiss LP: A member of the GAGE family of tumor antigens is an anti-apoptotic gene that confers resistance to Fas/CD95/APO-1, Interferon-gamma, taxol and gamma-irradiation. Cancer Biol Ther 1: 380-387, 2002.

25. Kular RK, Yehiely F, Kotlo KU, Cilensek ZM, Bedi R and Deiss LP: GAGE, an antiapoptotic protein binds and modulates the expression of nucleophosmin/B23 and interferon regulatory factor 1. J Interferon Cytokine Res 29: 645-655, 2009.

26. Zeng Y, He Y, Yang F, Mooney SM, Getzenberg RH, Orban J and Kulkarni P: The cancer/testis antigen prostate-associated gene 4 (PAGE4) is a highly intrinsically disordered protein. J Biol Chem 286: 13985-13994, 2011.

27. Israels ED and Israels LG: The cell cycle. Oncologist 5: 510-513, 2000.

28. Kim JK and Diehl JA: Nuclear cyclin D1: An oncogenic driver in human cancer. J Cell Physiol 220: 292-296, 2009.

29. Mahale S, Bharate SB, Manda S, Joshi P, Jenkins PR, Vishwakarma RA and Chaudhuri B: Antitumour potential of BPT: A dual inhibitor of cdk4 and tubulin polymerization. Cell Death Dis 6: e1743, 2015.

30. Lin Z, Xiong L, Zhou J, Wang J, Li Z, Hu H and Lin Q: $\gamma$-Glutamylcyclotransferase knockdown inhibits growth of lung cancer cells through G0/G1 phase arrest. Cancer Biother Radiopharm 30: 211-216, 2015.

31. Lv XJ, Zhao LJ, Hao YQ, Su ZZ, Li JY, Du YW and Zhang J: Schisandrin B inhibits the proliferation of human lung adenocarcinoma A549 cells by inducing cycle arrest and apoptosis. Int J Clin Exp Med 8: 6926-6936, 2015. 
32. Roy S, Gu M, Ramasamy K, Singh RP, Agarwal C, Siriwardana S, Sclafani RA and Agarwal R: p21/Cip1 and p27/Kip1 are essential molecular targets of inositol hexaphosphate for its antitumor efficacy against prostate cancer. Cancer Res 69: 1166-1173, 2009.

33. Perisanidis C, Perisanidis B, Wrba F, Brandstetter A, El Gazzar S, Papadogeorgakis N, Seemann R, Ewers R, Kyzas PA and Filipits M: Evaluation of immunohistochemical expression of p53, p21, p27, cyclin D1, and Ki67 in oral and oropharyngeal squamous cell carcinoma. J Oral Pathol Med 41: 40-46, 2012.

34. Zhang J, Yang Y, Lei L and Tian M: Rhizoma Paridis Saponins induces cell cycle arrest and apoptosis in non-small cell lung carcinoma A549 cells. Med Sci Monit 21: 2535-2541, 2015.
35. Horn HF and Vousden KH: Coping with stress: Multiple ways to activate p53. Oncogene 26: 1306-1316, 2007.

36. Chipuk JE and Green DR: Cytoplasmic p53: Bax and forward. Cell Cycle 3: 429-431, 2004.

37. Gjerstorff MF, Burns J and Ditzel HJ: Cancer-germline antigen vaccines and epigenetic enhancers: Future strategies for cancer treatment. Expert Opin Biol Ther 10: 1061-1075, 2010.

38. Simpson AJ, Caballero OL, Jungbluth A, Chen YT and Old LJ: Cancer/testis antigens, gametogenesis and cancer. Nat Rev Cancer 5: 615-625, 2005. 